intraoperative PCI is particularly measurable. With lesser PCI that would predict optimal surgical outcome, the preoperative PCI from a practical imaging would be useful as a predictive method to assess possibility of an optimal cytoreduction and the optimal time of surgery as well.

Poster (006)

Epithelial Ovarian Cancer including Borderline Tumor

https://doi.org/10.3802/jgo.2021.32.S1.006

\section{Correlation of CD47 expression and treatment response to neo-adjuvant chemotherapy in advanced epithelial ovarian cancer}

\section{P. Veena," Neha Loharkar, Durairaj Jayalakshmi, Nachiappa Ganesh Rajesh}

Jawaharlal Institute of Postgraduate Medical Education \& Research (JIPMER), Pondicherry, India (veenup_2001@yahoo.com)

Objective: To study the expression of CD47 and its correlation with treatment response to neo-adjuvant chemotherapy (NACT) among patients with advanced ovarian malignancy (CD47 may play an inhibitory role in NK cell-mediated cytotoxicity against cancer cells, implying a possible mechanism of immune escape in cancer cells leading to aggressive behavior. Overexpression of CD47 increases the risk of metastasis and is negatively correlated with prognosis).

Methods: In this hospital-based cohort study, 92 patients diagnosed with advanced ovarian cancer decided for NACT were recruited. Baseline clinical data, including cancer antigen 125 levels and contrast enhanced computed tomography findings, were noted. These women were subjected to image-guided fine needle aspiration cytology of the ovarian tumor for pathological diagnosis. We prepared cell blocks for immunohistochemistry and studied CD47 expression. After 3 cycles of NACT, patients were assessed for treatment response. Among patients with good treatment response and deemed to be operable after 3 cycles of NACT, interval cytoreductive surgery was performed. The level of cytoreduction was studied among these patients and was correlated with CD47 levels.

Results: Out of 92 patients, 61 were assessed to have a good response to NACT after 3 cycles and underwent interval cytoreduction. Among these, six patients had suboptimal cytoreduction. Around 19 (86.5\%) women with high CD47 expression and 36 (92.6\%) women with low CD47 expression had optimal cytoreduction (R0 and R1). But the difference is statistically insignificant. Out of 36 women with high CD47 expression, 17 (47.2\%) women had a poor response compared to $19(52.8 \%)$ women who had a good response. Around two-thirds of the patients with low CD47 expression had a good response to NACT. But the difference is statistically insignificant. Conclusion: The $39.1 \%$ of patients had high CD47 expression in our study, much lesser than reported in the published literature. There was no significant association between CD47 expression and response to NACT in patients with advanced epithelial ovarian malignancy in our study.

Poster (007)

Basic/Translational Science

https://doi.org/10.3802/jgo.2021.32.S1.007

\section{Digital spatial profiling of metastatic clear cell carcinoma reveals intra-tumor heterogeneity in epithelial-mesenchymal gradient}

Duncan Yi-Te Wang ${ }^{1}$ Tuan Zea Tan ${ }^{2}$ Ya-Ting Tai ${ }^{1}$ Jieru Ye, Wei-Chou Lin, ${ }^{3}$ Lin-Hung Wei, ${ }^{4}$ Ruby Yun-Ju Huang ${ }^{1, *}$

'School of Medicine, College of Medicine, National Taiwan University, Taipei, Taiwan (rubyhuang@ntu.edu.tw)

${ }^{2}$ Cancer Science Institute of Singapore, National University of Singapore, Singapore

${ }^{3}$ Department of Pathology, National Taiwan University Hospital, Taipei, Taiwan

${ }^{4}$ Department of Obstetrics \& Gynecology, College of Medicine, National Taiwan University, Taipei, Taiwan

Objective: Advanced ovarian clear cell carcinoma (OCCC), known to harbor intra-tumor heterogeneity (ITH), is chemoresistant and poor prognostic, possessing distinct molecular and histological characteristics. However, detailed spatial information of the nature of ITH within OCCC remains unclear. Here, we aim to decipher the hidden spatial information within the tumor samples of primary (Pri) and colonic metastatic (Met) sites from one advanced OCCC patient and build up a geospatial analysis pipeline for further exploratory study.

Methods: NanoString Digital spatial profiling (DSP) GeoMx platform was utilized to perform multiplex protein expression analysis on OCCC samples, and 18 to 19 regions of interest (ROIs) were selected per sample. Tumor cells were classified into different subgroups based on the expression patterns from the heatmap of the clustered data.

Results: The $47.6 \%$ of the PanCK-positive segments of ROIs from Met were clustered into C2_tumor, while those from Pri were mostly clustered into C1_tumor, suggesting that Met were more heterogeneous ( $\chi^{2}$ test, $\mathrm{p}=0.00371$ ). For tumor cells from different subgroups in Met, the difference between the expression levels of PanCK, SMA, Ki-67, FN, NCAM and ITGAX were statistically significant, which reflected the existence of an epithelial-mesenchymal (EM) gradient within Met (1-way 
analysis of variance, $\mathrm{p}<0.05)$.

Conclusion: The spatial resolution revealed an EM gradient within Met but not Pri. This provides an unprecedented view of the EM gradient during the progression of cancer such as OCCC. Our study provides the first spatially resolved in situ evidence of intermediate or hybrid EM states within the tumor samples of similar morphology.

Poster (008)

Epithelial Ovarian Cancer including Borderline Tumor

https://doi.org/10.3802/jgo.2021.32.\$1.008

\section{DKK3, down-regulated in invasive epithelial ovarian cancer was associated with chemoresistance and enhanced paclitaxel susceptibility via inhibition of $\beta$-catenin-P- glycoprotein pathway}

\author{
Que Thanh Thanh Nguyen, ${ }^{1}$ Kyung-Mi Choi, ${ }^{2}$ Tae Jin Lee, ${ }^{3}$ \\ So Young Kim, ${ }^{2}$ Jae Hyung Kim, ${ }^{4}$ Eun-Ju Lee, ${ }^{2, *}$ \\ 'Chung-Ang University, Seoul, Korea \\ ${ }^{2}$ Department of Obstetrics and Gynecology, Chung-Ang University School \\ of Medicine, Seoul, Korea (ejlee@cau.ac.kr) \\ ${ }^{3}$ Department of Pathology, Chung-Ang University School of Medicine, \\ Seoul, Korea \\ ${ }^{4}$ Department of Radiology, Sanggye Paik Hospital, Inje University College of \\ Medicine, Seoul, Korea
}

Objective: This study aimed to assess aberrant Dickkopf-3 (DKK3) expression in epithelial ovarian carcinoma and to determine the therapeutic role of DKK3 in ovarian cancer. Methods: DKK3 protein expression was examined by immunohistochemistry using tissue blocks from 82 patients with invasive ovarian adenocarcinoma, and 15 normal, 19 benign, and 10 borderline tumors as controls. Survival data were estimated using Kaplan-Meier estimates, and multivariate analysis was performed using the Cox regression method. Paclitaxel-resistant cell lines were manufactured with TOV-21G and OV-90 ovarian cancer cell lines. The protein expression and cell viability were assessed by Western blotting and MTT assay, respectively.

Results: DKK3 was significantly down-regulated in invasive carcinoma compared to normal, benign, and borderline tumors. Univariate analysis showed that higher FIGO stage, DKK3 loss, suboptimal debulking operation, and chemo-resistance were significantly associated with low disease-free survival of serous carcinoma patients. DKK3 loss occurred in $56.1 \%$ invasive carcinoma cases and was significantly associated with chemoresistance in serous adeno carcinoma $(\mathrm{p}=0.029)$. Paclitaxelresistant cell lines were successfully established. Western blot analysis showed that DKK3 was lost in paclitaxel-resistant cells and as expected, $\beta$-catenin and P-glycoprotein were upregulated. Secreted DKK3 exerted an anti-proliferative effect and induced paclitaxel susceptibility in paclitaxel-resistant cells $(\mathrm{p}<0.01)$. Secreted DKK3 was internalized into cells, reduced $\beta$-catenin activity, hence inhibited P-glycoprotein, suggesting an axis link between them.

Conclusion: DKK3 loss was frequent in invasive epithelial ovarian cancer and significantly associated with chemoresistance. Secreted DKK3 has an anti-proliferative effect on paclitaxel-resistant cells by inhibiting $\beta$-catenin and P-glycoprotein, suggesting that DKK3 could be therapeutics targeting paclitaxel-resistant ovarian cancer.

Poster (009)

Conservative \& Fertility Preservation

https://doi.org/10.3802/jgo.2021.32.\$1.009

\section{Fertility sparing surgery in malignant ovarian germ cell tumors (MOGCTs): 15 years experiences}

\author{
Shima Alizadeh, " Narges Zamani, Mohadese Rezaei Poor, \\ Sedigheh Ghasemian Dizajmehr, Mitra Modares Gilani \\ Tehran University of Medical Sciences, Tehran, Iran \\ (alizadeh.shima@gmail.com)
}

Objective: Our aim is to evaluate the reproductive outcome of fertility-sparing surgery and chemotherapy among young women diagnosed with malignant ovarian germ cell tumor (MOGCT) of any stage.

Methods: In current retrospective study we evaluated 79 patients with MOGCT whom visited at Imam Center, Vali-Asr Hospital, Gynecology Oncology department during 2001-2016. Reproductive outcomes (menstruation status \& childbearing) followed fertility preserving surgery and adjuvant chemotherapy by filling questionnaires. Statistical analysis was done with SPSS software, $\chi^{2}$ tests were done, and significance determined at $\mathrm{p} \leq 0.05$.

Results: Among 79 young women who underwent fertilitysparing treatment,72 patients followed up for reproductive outcome and 7 patients excluded due to death ( 3 cases), XY genotyping ( 3 cases), and bilateral ovarian involvement (1 case). The mean age at presentation was 23 years (range $=19-33$ years). The 5 and 10-year disease-free survival rate was $87 \%$ and $94.4 \%$, respectively. The overall survival rate was $94.4 \%$ at 5 and 10 years. Regular menstruation recovered in 60 of 72 patients after treatment $(83 \%)$. All patients without adjuvant chemotherapy experienced regular menstruation while normal menstruation retrieved in $78 \%$ in adjuvant chemotherapy group at the end of treatment. This retrieval of regular menstruation was not 